H. Thermann et al.

\title{
Die Frakturen des kindlichen Fußes „Was wächst sich aus"?
}

\author{
$N_{i}$ \\ immt man einen kindlichen Fuß zur \\ Untersuchung in die Hand, merkt man \\ sofort:
}

Dieser Fuß ist anders!

Die anatomischen Unterschiede zum Erwachsenen bestehen aus Besonderheiten im Hinblick auf Größe, Achsen sowie Knochen-, Knorpel-und Bandstrukturen. Das Längenwachstum des Fußes findet, verglichen zum übrigen Skelett, wesentlich schneller statt. So ist nach Untersuchungen von BLISS et al. ein wesentlicher Anteil des Längenwachstums bereits bis zum 5. Lebensjahr abgeschlossen. Bei 1-jährigen Mädchen bzw. $1^{1} /{ }_{2}$-jährigen Jungen hat der Fuß bereits $50 \%$ seiner Gesamtlänge erreicht, wohingegen dies beim Femur erst nach 3 Jahren der Fall ist. Obwohl dieses Wachstumspotential besonders bei den Fußknochen mit Epiphyse besteht, ist es im Gegensatz zu den langen Röhrenknochen schon mit dem 12. Lebensjahr weitgehend erschöpft. Dies ist bedeutungsvoll für die einzuschlagende Behandlungsstrategie. Wo steht die Behandlung der Frakturen des kindlichen Fußes 1997? Welche Frakturen können konservativ, welche müssen operativ behandelt werden? Anhand des eigenen Krankengutes sowie den Berichten aus der Literatur soll in der folgenden Arbeit Behandlungsvorschläge gemacht wer- den, die zum einen die Ergebnisse der Literatur berücksichtigen, zum andern aber auch neuere Therapiekonzepte und operative Techniken vorstellen. Im einzelnen führen Frakturen und Luxationen des Talus, Calcaneus, der Chopartund Lisfrancreihe zu schweren Gelenkverwerfungen, welche auch beim Kleinkind ohne entsprechende Reposition durch das Wachstumspotential nicht ausgeglichen werden können. Die Fortschritte der „Erwachsenenchirurgie“ am Fuß haben sich auch in der „Kinderchirurgie" niedergeschlagen, so daß die komplizierte Fraktur des kindlichen Fußes nicht mehr die alleinige Domäne der konservativen Behandlung sein kann. Die Verbesserung der Versorgung von komplizierten Frakturen des kindlichen Fußes muß ein weiterer Fokus in der zunehmenden Diskussion über die Behandlung von Verletzungen des Fußes sein. Die wissenschaftlich exakte Aufarbeitung der Frakturen des kindlichen Fußes steht vor dem Problem, daß bislang zu geringe Fallzahlen evaluiert wurden: Um detaillierte Therapiekonzepte einzelner seltener Problemfrakturen (z. B. Talus-, Calcaneusfrakturen) wissenschaftlich fundiert überprüfen $z u$ können, bedarf es multizentrischer Studien mit standardisierten Protokollen.
H. Thermann
Prof. H. Tscherne 